\title{
The Möbius function of the permutation pattern poset
}

\author{
Einar Steingrímsson* And Bridget Eileen Tenner
}

\begin{abstract}
A permutation $\tau$ contains another permutation $\sigma$ as a pattern if $\tau$ has a subsequence whose elements are in the same order with respect to size as the elements in $\sigma$. This defines a partial order on the set of all permutations, and gives a graded poset $\mathcal{P}$. We give a large class of pairs of permutations whose intervals in $\mathcal{P}$ have Möbius function 0. Also, we give a solution to the problem when $\sigma$ occurs precisely once in $\tau$, and $\sigma$ and $\tau$ satisfy certain further conditions, in which case the Möbius function is shown to be either $-1,0$ or 1 . We conjecture that for intervals $[\sigma, \tau]$ consisting of permutations avoiding the pattern 132, the magnitude of the Möbius function is bounded by the number of occurrences of $\sigma$ in $\tau$. We also conjecture that the Möbius function of the interval $[1, \tau]$ is $-1,0$ or 1 .

AMS 2000 subJeCt Classifications: Primary 05A05; Secondary 06A07, 37F20.

KEYWORDS AND PHRASES: Möbius function, permutation, permutation pattern, poset.
\end{abstract}

\section{Introduction}

In this paper, permutations are of the letters $1,2, \ldots, n$ (for various $n$ ) and a pattern in a permutation $\pi$ is a subsequence in $\pi$, the relative sizes of whose elements come in some prescribed order. For example, a 123-pattern is simply an increasing subsequence of length three. The permutation 246153 has two occurrences of the pattern 123, namely the subsequences $\langle 246\rangle$ and $\langle 245\rangle$. The permutation 246153 also has one occurrence of the pattern 3142, namely the subsequence $\langle 4153\rangle$. It is easy to see that the set of all permutations (of arbitrary, positive, numbers of letters) forms a graded poset $\mathcal{P}$ with respect to pattern containment. That is, a permutation $\sigma$ is smaller than another permutation $\tau$ in $\mathcal{P}$ if $\sigma$ occurs as a pattern in $\tau$.

${ }^{*}$ The first author was supported by grants no. 060005013 and 090038011 from the Icelandic Research Fund. 
A classical problem for any poset, first explicitly mentioned by Wilf [5] in this particular case, is to understand its Möbius function $\mu$. The first result so far in this direction was given by Sagan and Vatter [3], who solved the problem in the case of layered permutations. They noted that the poset of layered permutations is isomorphic to a certain poset of compositions of an integer, and they gave a formula for computing the Möbius function of that poset. A permutation is layered if it is the concatenation of decreasing sequences, where the letters in each sequence are smaller than all letters in later sequences. An example of such a permutation is 321546987 .

Looking at examples of the Möbius function of intervals in the pattern poset, it is easy to infer that this is hard to understand in the general case. In particular, the Möbius function for this poset does not alternate in sign with rank, which is a discouraging starting point.

In this paper, we present some results for this problem. Most of our results stem from looking at particular occurrences of a pattern $\sigma$ in a permutation $\tau$ and analyzing the complement in $\tau$ of an occurrence. In particular, in Section 3, we give a large class of pairs of permutations $\sigma$ and $\tau$ for which the Möbius function of the interval $[\sigma, \tau]$ is 0 (Theorem 3.2). These intervals occur when there is a sequence of consecutive letters of $\tau$ that are not in any occurrence of $\sigma$, and which form an interval of values $\{a, a+1, \ldots, a+b\}$ for some $a$ and some $b \geq 1$. One such pair is $(132,859324617)$ : no letter of the contiguous subsequence 324 belongs to any occurrence of the pattern 132 in 859324617 .

In Section 4, we solve the problem in the case where $\sigma$ occurs precisely once in $\tau$ and the complement of $\sigma$ in $\tau$ satisfies certain conditions. The Möbius function in this case is either -1 , 0 , or 1 (Corollary 4.12). This follows from a more general result (Theorem 4.11) about a particular kind of fixed occurrence of $\sigma$ in $\tau$. We believe that there are other interesting classes of intervals also having Möbius function -1, 0 or 1, as we explain below.

In the final section, we conjecture that if the permutations in an interval $[\sigma, \tau]$ avoid the pattern 132 (equivalently, if $\tau$ avoids 132 ), then the absolute value of the Möbius function of $[\sigma, \tau]$ is bounded by the number of occurrences of $\sigma$ in $\tau$. Due to the symmetries among 132, 231, 213 and 312, the pattern 132 can be replaced by any one of the other three patterns in this conjecture. Thus, this conjecture is equivalent to the conjecture that if the absolute value of the Möbius function of an interval $[\sigma, \tau]$ exceeds the number of occurrences of $\sigma$ in $\tau$ then $\tau$ must contain all of the patterns 132, 231, 213 and 312. Observe also that if this conjecture holds, then, for 
132-avoiding intervals $[\sigma, \tau]$ where $\sigma$ occurs precisely once in $\tau$, the Möbius function must be 0,1 or -1 .

Finally, we conjecture that if a permutation $\tau$ avoids 132, then the interval $[1, \tau]$ has Möbius function $-1,0$ or 1 .

\section{Definitions and notation}

Let $\mathcal{S}_{n}$ be the set of permutations of the letters $\{1,2, \ldots, n\}$. We represent permutations in one-line notation, meaning that the permutation $\sigma \in \mathcal{S}_{k}$ is denoted $\sigma=\sigma(1) \sigma(2) \cdots \sigma(k)$. Throughout this section, fix permutations $\sigma \in \mathcal{S}_{k}$ and $\tau \in \mathcal{S}_{n}$, with $k \leq n$.

Definition 2.1. If $\pi$ is a permutation of a set of $k$ integers, then its standard form is the permutation of $\{1,2, \ldots, k\}$ whose letters are in the same relative order of size as those of $\pi$. We say that two permutations are order isomorphic if they have the same standard form.

For example, the standard form of both 3615 and 4725 is 2413 , meaning that 3615,4725 , and 2413 are all order isomorphic to each other.

Definition 2.2. The permutation $\tau$ contains $\sigma$ (also, $\tau$ has a $\sigma$-pattern) if there exist indices $i_{1}<\cdots<i_{k}$ such that $\sigma$ is order isomorphic to $\tau\left(i_{1}\right) \cdots \tau\left(i_{k}\right)$. If $\tau$ does not contain $\sigma$, then $\tau$ avoids $\sigma$.

If $\tau$ has a $\sigma$-pattern, with $i_{1}<\cdots<i_{k}$ as in Definition 2.2, then $\tau\left(i_{1}\right) \cdots \tau\left(i_{k}\right)$ is an occurrence of $\sigma$ in $\tau$. The substring $\tau\left(i_{j_{1}}\right) \cdots \tau\left(i_{j_{e}}\right)$ will be denoted $\left\langle\sigma\left(j_{1}\right) \cdots \sigma\left(j_{\ell}\right)\right\rangle$, and occurrences of $\sigma$ will be distinguished by subscripts: $\langle\sigma\rangle_{i}$. When speaking of an occurrence $\langle\sigma\rangle$ it is necessary to know in what larger permutation this occurrence sits ( $\tau$ in the current discussion). In many instances this will be clear from the context, and will not be specified further.

Example 2.3. Let $\tau=74136825$ and $\sigma=1243$. Then $\langle 1365\rangle$ and $\langle 1385\rangle$ are the only two occurrences of $\sigma$ in $\tau$. We can name $\langle\sigma\rangle_{1}=\langle 1365\rangle$ and $\langle\sigma\rangle_{2}=\langle 1385\rangle$.

If $\tau$ has a $\sigma$-pattern, and $\langle\sigma\rangle$ is a particular occurrence of $\sigma$ in $\tau$ with $x \in \tau \backslash\langle\sigma\rangle$ a letter of $\tau$ not in $\langle\sigma\rangle$, then we will simplify notation somewhat and write

$$
\langle\sigma\rangle+x:=\langle\langle\sigma\rangle \cup\{x\}\rangle
$$

to indicate the occurrence of a pattern in $\tau$ (different from the $\sigma$-pattern) formed by the letters $\langle\sigma\rangle \cup\{x\}$. The notation $\langle\sigma\rangle+S$ is analogously defined, whenever $S$ is a subset of letters in $\tau \backslash\langle\sigma\rangle$. 
Example 2.4. With the notation of Example 2.3, $\langle\sigma\rangle_{1}+2=\langle 13625\rangle$ and $\langle\sigma\rangle_{1}+7=\langle 71365\rangle$.

Definition 2.5. If $\tau$ contains $\sigma$, then write $\sigma \leq \tau$. If $\sigma \leq \tau$, but $\sigma$ and $\tau$ are not order isomorphic (in particular, $\sigma \neq \tau$ ), then write $\sigma<\tau$. If $\sigma<\tau$ and $k=n-1$, then $\tau$ covers $\sigma$. Let $\mathcal{P}$ denote the poset of all permutations of arbitrary, positive, numbers of letters, ordered by pattern containment. That is, the partial ordering on $\mathcal{P}$ is defined by the relation $\leq$.

Two elements $s$ and $t$ in a poset $P$ determine an interval $[s, t]=\{x \in$ $P \mid s \leq x \leq t\}$, with $[s, t]=\emptyset$ if $s \not \leq t$ in $P$. We also define the half-open interval $[s, t)=\{x \in P \mid s \leq x<t\}$.

It is easy to see that the permutation pattern poset $\mathcal{P}$ is graded. That is, the lengths of all maximal chains in an interval are the same. More precisely, the length of a maximal chain between two permutations $\sigma$ and $\tau$, where $\sigma \leq \tau$, is the difference between the number of letters of $\tau$ and of $\sigma$. This number is also called the rank of the interval.

Definition 2.6. Given an interval $[s, t]$ in a poset $P$, the Möbius function $\mu_{P}$ of this interval, written $\mu$ when no confusion will arise, is recursively defined by

$$
\mu(s, t)= \begin{cases}0 & \text { if } s \not \leq t, \\ 1 & \text { if } s=t, \text { and } \\ -\sum_{s \leq x<t} \mu(s, x) & \text { otherwise. }\end{cases}
$$

From Definition 2.6, it is clear that if $s \neq t$, then the sum of the values $\mu(s, x)$ over all $x$ in the interval $[s, t]$ equals zero. For an example of how the Möbius function can be computed from Definition 2.6, see Figure 1.

Definition 2.7. We regard patterns (permutations) $\sigma$ in the permutation pattern poset $\mathcal{P}$ as functions from $\mathcal{P}$ to $\mathbb{N}$, where $\sigma(\tau)$ is the number of occurrences of $\sigma$ in $\tau$.

Note that if $\sigma \not \leq \tau$, then $\sigma(\tau)=0$.

Example 2.8. Let $\sigma=231$ and $\tau=23541$. Then $\sigma(\tau)=5$ because there are five distinct occurrences of the pattern $\sigma$ in $\tau:\langle 231\rangle,\langle 251\rangle,\langle 241\rangle,\langle 351\rangle$, and $\langle 341\rangle$.

Definition 2.9. If a permutation $\tau$ maps a non-singleton interval onto an interval, then this image is an interval block. More precisely, fix an integer $b \geq 1$. If $\tau$ has a factor (consecutive substring) $I=\tau(a) \tau(a+1) \cdots \tau(a+b)$, 


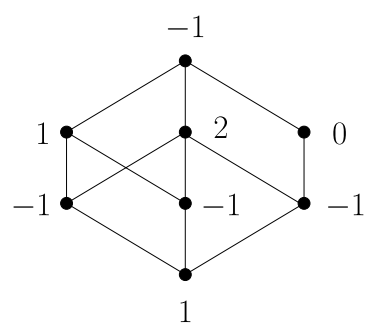

Figure 1: Computing the Möbius function of an interval: the number at each element in this poset gives the value of the Möbius function of the interval from the minimum element to that element.

where the set of values $\{\tau(a), \tau(a+1), \ldots, \tau(a+b)\}$ consists of all the numbers $a^{\prime}, a^{\prime}+1, \ldots, a^{\prime}+b$ for some $a^{\prime}$, then $I$ is an interval block.

Example 2.10. The permutation 71342865 has interval blocks 34, 342, 1342,65 and 71342865 .

This definition may bring to mind simple permutations: $\pi$ is simple if it has no interval blocks other than $\pi$ itself. For information about these permutations, see [2] and [1].

Definition 2.11. Suppose that $\sigma \leq \tau$ in $\mathcal{P}$, and fix an occurrence $\langle\sigma\rangle$ of $\sigma$ in $\tau$. If $\tau$ has an interval block that does not intersect the occurrence $\langle\sigma\rangle$, then the pair $(\langle\sigma\rangle, \tau)$ has an interval block. Otherwise the pair is interval free.

Definition 2.12. Suppose that $\sigma \leq \tau$. If $\tau$ has an interval block that does not intersect any occurrence of $\sigma$, then the pair $(\sigma, \tau)$ has an interval block.

Example 2.13. Let $\sigma=2341$ and $\tau=162395784$. The pair $(\sigma, \tau)$ has interval block $I=23$ because the occurrences of $\sigma$ in $\tau$, namely $\langle 6784\rangle$ and $\langle 5784\rangle$, are each disjoint from $I$.

To compute the Möbius function $\mu(\sigma, \tau)$, one examines the interval $[\sigma, \tau] \subset \mathcal{P}$. For our purposes, it will be helpful first to examine a slightly different poset, described below.

Definition 2.14. Suppose that $\sigma \leq \tau$, and that $\langle\sigma\rangle$ is a particular occurrence of $\sigma$ in $\tau$. Set $C:=\tau \backslash\langle\sigma\rangle$ to be the complement of $\langle\sigma\rangle$ in $\tau$. Let the occurrence poset $[\langle\sigma\rangle, \tau]$ denote the partially ordered set consisting of permutations formed by deleting arbitrary subsets of letters of $C$ from $\tau$. Two such permutations are considered equivalent if they are order isomorphic and if the specified occurrence $\langle\sigma\rangle$ is in the same positions in each. A 


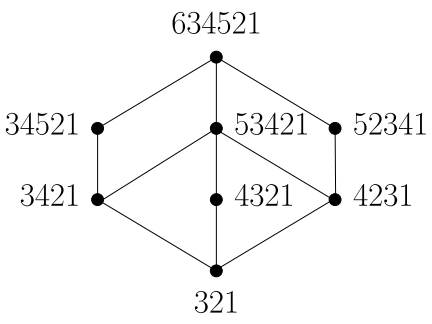

$[321,634521]$

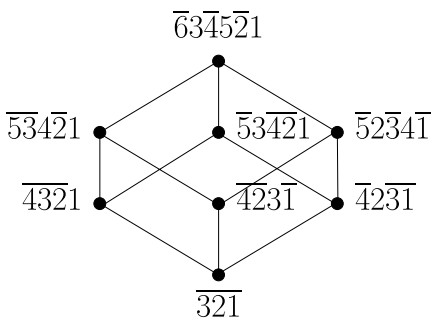

$[\overline{321}, \overline{6} 3 \overline{4} 5 \overline{2} 1]$

Figure 2: The posets $[\sigma, \tau]$ and $[\langle\sigma\rangle, \tau]$, with $\sigma=321, \tau=634521$, and $\langle\sigma\rangle=\langle 642\rangle$. In the latter poset, the letters in the occurrence $\langle\sigma\rangle$ are marked.

permutation $\rho$ is covered by $\pi$ if $\rho$ can be obtained from $\pi$ by removing a letter of $\pi \backslash\langle\sigma\rangle$. This defines the partial ordering in $[\langle\sigma\rangle, \tau]$.

In particular, if $\sigma(\tau)=1$, then $[\langle\sigma\rangle, \tau]=[\sigma, \tau]$. If $\tau=\overline{6} 3 \overline{4} 5 \overline{2} 1$, where we have overlined the letters in a particular occurrence $\langle\sigma\rangle$ of the pattern 321 , then the permutations obtained by deleting any single letter of $C=\{3,5,1\}$ are $\overline{53} 4 \overline{2} 1, \overline{5} 3 \overline{42} 1$ and $\overline{5} 2 \overline{3} 4 \overline{1}$. Note that the first two of these are identical as permutations, but are considered distinct here because $\langle\sigma\rangle$ is in different positions. Figure 2 depicts the intervals $[\sigma, \tau]$ and $[\langle\sigma\rangle, \tau]$ for this example.

When no confusion will arise, the Möbius function of the interval $[\langle\sigma\rangle, \tau]$ will be denoted

$$
\mu(\langle\sigma\rangle, \tau):=\mu_{[\langle\sigma\rangle, \tau]}(\langle\sigma\rangle, \tau) .
$$

Definition 2.14 will be helpful for analyzing the Möbius function of a generic interval $[\sigma, \tau]$ in $\mathcal{P}$ because the Möbius function can be computed as an alternating sum of chains according to length. In any chain from $\tau$ to $\sigma$ in $\mathcal{P}$, the final element must be a $\sigma$-pattern. Thus this chain highlights a particular occurrence of $\sigma$ in $\tau$ and removes the other letters successively. Of course, we must exercise caution because there may be some overcounting of these chains. One instance in which there can be no overcounting is when $\sigma(\tau)=1$, and hence $[\sigma, \tau]=[\langle\sigma\rangle, \tau]$.

\section{Pairs with interval blocks}

Throughout this section, fix $\sigma \in \mathcal{S}_{k}$ and $\tau \in \mathcal{S}_{n}$ such that $\sigma \leq \tau$ in $\mathcal{P}$, and fix an occurrence $\langle\sigma\rangle$ of $\sigma$ in $\tau$. 
Theorem 3.1. Let $\sigma \in \mathcal{S}_{k}$ and $\tau \in \mathcal{S}_{n}$, with $\sigma \leq \tau$. If the pair $(\langle\sigma\rangle, \tau)$ has an interval block, then

$$
\mu(\langle\sigma\rangle, \tau)=0 .
$$

Proof. We prove the theorem by induction on $n-k$, the rank of the interval $[\langle\sigma\rangle, \tau]$. Since $(\langle\sigma\rangle, \tau)$ has an interval block, $n-k$ must be at least 2 . In the case $n-k=2$, which serves as the basis of the induction, the pair $(\langle\sigma\rangle, \tau)$ has precisely one interval block (with two elements), which we call $I$. This implies that the poset $[\langle\sigma\rangle, \tau]$ is a three element chain, where the middle element is obtained by removing one of the two letters of $I$ from $\tau$. The Möbius function of such a chain is 0 , as claimed.

Assume that the result holds for all intervals of rank less than $n-k$, where $n-k \geq 3$. Let $I$ be an interval block in the pair $(\langle\sigma\rangle, \tau)$, where $[\langle\sigma\rangle, \tau]$ has rank $n-k$. Let $\pi$ be the permutation obtained from $\tau$ by deleting all but one letter from $I$, but otherwise leaving $\tau$ intact. For each permutation $\rho \in[\langle\sigma\rangle, \tau]$ such that $\rho \not \leq \pi$, the pair $(\langle\sigma\rangle, \rho)$ must contain an interval block because $\rho$ must contain at least two letters originating from $I$.

Because $[\langle\sigma\rangle, \pi]$ is a closed interval in $[\langle\sigma\rangle, \tau]$, the sum of the values of the Möbius function over this interval is zero. Thus

$$
\begin{aligned}
\mu(\langle\sigma\rangle, \tau) & =-\sum_{\rho \in[\langle\sigma\rangle, \tau)} \mu(\langle\sigma\rangle, \rho) \\
& =-\sum_{\rho \in[\langle\sigma\rangle, \pi]} \mu(\langle\sigma\rangle, \rho)-\sum_{\substack{\rho \in[\langle\sigma\rangle, \tau) \\
\rho \notin \pi}} \mu(\langle\sigma\rangle, \rho) \\
& =-\sum_{\substack{\rho \in[\langle\sigma\rangle, \tau) \\
\rho \notin \pi}} \mu(\langle\sigma\rangle, \rho) .
\end{aligned}
$$

Now, each $\rho$ in the summation in Equation (3) is strictly smaller than $\tau$, so the rank of $[\langle\sigma\rangle, \rho]$ for each such $\rho$ is strictly less than $n-k$. Moreover, the pair $(\langle\sigma\rangle, \rho)$ contains an interval block. Hence, by the inductive hypothesis, $\mu(\langle\sigma\rangle, \rho)=0$ for all such $\rho$, which implies that $\mu(\langle\sigma\rangle, \tau)=0$, completing the proof.

As discussed earlier, the poset $[\langle\sigma\rangle, \tau]$ will be a helpful tool for the analysis of intervals in $\mathcal{P}$. In fact, Theorem 3.1 can be translated readily into a statement about intervals in $\mathcal{P}$, as shown in the following theorem. The proof of this result is entirely analogous to that of the previous theorem.

Theorem 3.2. If the pair $(\sigma, \tau)$ has an interval block, then

$$
\mu(\sigma, \tau)=0 .
$$


Proof. Consider $\sigma \in \mathcal{S}_{k}$ and $\tau \in \mathcal{S}_{n}$, with $\sigma \leq \tau$ in $\mathcal{P}$.

We prove the theorem by induction on $n-k$, the rank of the interval $[\sigma, \tau]$. Since $(\sigma, \tau)$ has an interval block, $n-k$ must be at least 2 . In the case $n-k=2$, which serves as the basis of the induction, the pair $(\sigma, \tau)$ as precisely one interval block (with two elements), which we call $I$. This implies that the poset $[\sigma, \tau]$ is a three element chain, where the middle element is obtained by removing one of the two letters of $I$ from $\tau$. The Möbius function of such a chain is 0 , as desired.

Assume that the result holds for all intervals of rank less than $n-k$, where $n-k \geq 3$. Let $I$ be an interval block in the pair $(\sigma, \tau)$, where $[\sigma, \tau]$ has rank $n-k$. Let $\pi$ be the permutation obtained from $\tau$ by deleting all but one letter from $I$, but otherwise leaving $\tau$ intact. For each permutation $\rho \in[\sigma, \tau]$ such that $\rho \not \leq \pi$, the pair $(\sigma, \rho)$ must contain an interval block because $\rho$ must contain at least two letters originating from $I$.

Because $[\sigma, \pi]$ is a closed interval in $[\sigma, \tau]$, the sum of the values of the Möbius function over this interval is zero. Thus

$$
\begin{aligned}
\mu(\sigma, \tau) & =-\sum_{\rho \in[\sigma, \tau)} \mu(\sigma, \rho) \\
& =-\sum_{\rho \in[\sigma, \pi]} \mu(\sigma, \rho)-\sum_{\substack{\rho \in[\sigma, \tau) \\
\rho \notin \pi}} \mu(\sigma, \rho) \\
& =-\sum_{\substack{\rho \in[\sigma, \tau) \\
\rho \notin \mathbb{Z} \pi}} \mu(\sigma, \rho) .
\end{aligned}
$$

Now, each $\rho$ in the summation in Equation (4) is strictly smaller than $\tau$, so the rank of $[\sigma, \rho]$ for each such $\rho$ is strictly less than $n-k$. Moreover, the pair $(\sigma, \rho)$ contains an interval block. Hence, by the inductive hypothesis, $\mu(\sigma, \rho)=0$ for all such $\rho$, which implies that $\mu(\sigma, \tau)=0$, completing the proof.

There are several things to note about Theorem 3.2. First, the result does not hold if there is an interval block in $\tau$ that is disjoint from some, but not all, occurrences of $\sigma$ in $\tau$. For example, let $\sigma=12$ and $\tau=3412$. The interval block 12 is disjoint from the occurrence $\langle\sigma\rangle_{1}=\langle 34\rangle$, but not from the occurrence $\langle\sigma\rangle_{2}=\langle 12\rangle$. However, if one considers the poset $\left[\langle\sigma\rangle_{1}, \tau\right]$, then $\mu_{\left[\langle\sigma\rangle_{1}, \tau\right]}\left(\langle\sigma\rangle_{1}, \tau\right)=0$, whereas $\mu_{[\sigma, \tau]}(\sigma, \tau)=1$.

Also, the converse of Theorem 3.2 is false. For example, let $\sigma=1$ and $\tau=123$. The pair $(\sigma, \tau)$ does not have an interval block, but $\mu(\sigma, \tau)=0$. Thus, the property $\mu(\sigma, \tau)=0$ cannot be characterized completely by the presence of an interval block. 


\section{Intervals describing a fixed occurrence}

Throughout this section, fix $\sigma \in \mathcal{S}_{k}$ and $\tau \in \mathcal{S}_{n}$ such that $\sigma \leq \tau$ in $\mathcal{P}$, and fix an occurrence $\langle\sigma\rangle$ of $\sigma$ in $\tau$.

In this section we give two results showing that the Möbius function of a class of intervals is 1 or -1 , the sign depending on only on the rank of the interval. The first of these, Theorem 4.4, deals with intervals that are boolean algebras. That, in turn, is the basis for an extension to a more general result in Corollary 4.12.

Definition 4.1. Let $\langle\sigma\rangle$ be an occurrence of $\sigma$ in $\tau$. A region in $\tau$ (with respect to $\sigma$ ) is a maximal consecutive substring of the complement of $\langle\sigma\rangle$ in $\tau$.

We now define a quality of a pair $(\langle\sigma\rangle, \tau)$, which informally means that every pair of distinct letters in $\tau \backslash \sigma$ is separated either in value or in position by some letter of $\langle\sigma\rangle$. This quality is defined more precisely as follows.

Definition 4.2. The pair $(\langle\sigma\rangle, \tau)$ is separated if for all $x<y$ that are in the same region of $\tau \backslash\langle\sigma\rangle$, there exists a $j$ such that $x<\langle\sigma(j)\rangle<y$.

Example 4.3. The pair $(\langle\sigma\rangle, \tau)=(\langle 653\rangle, 146253)$ is separated: although 1 and 4 lie in the same region of $\tau \backslash\langle\sigma\rangle$, we have $1<\langle\sigma(1)\rangle=\langle 3\rangle<4$.

The pair $(\langle\sigma\rangle, \tau)=(\langle 764\rangle, 1357264)$ is not separated because 1 and 3 belong to the same region of $\tau \backslash\langle\sigma\rangle$, but the only letter between them in value, the 2 , does not lie in $\langle\sigma\rangle$.

Theorem 4.4. The interval $[\langle\sigma\rangle, \tau]$ is boolean if and only if the pair $(\langle\sigma\rangle, \tau)$ is separated. In this case, therefore, $\mu(\langle\sigma\rangle, \tau)=(-1)^{n-k}$, where $k$ and $n$ are the ranks of $\sigma$ and $\tau$, respectively.

Proof. The only letters that can be deleted from $\tau$ in the interval $[\langle\sigma\rangle, \tau]$ are elements of $\tau \backslash\langle\sigma\rangle$, since letters of the occurrence $\langle\sigma\rangle$ must be intact in each element of the interval. Suppose that $\sigma \in \mathcal{S}_{k}$ and $\tau \in \mathcal{S}_{n}$, so $[\sigma, \tau]$ has rank $n-k$. It is boolean if and only if the letters of $\tau \backslash\langle\sigma\rangle$ can be removed in any order, always yielding distinct permutations. This happens if and only if at no point are there letters $x$ and $x+1$ adjacent in $\rho \backslash\langle\sigma\rangle$ for some $\rho \in[\langle\sigma\rangle, \tau]$. This is equivalent to the pair $(\langle\sigma\rangle, \tau)$ being separated.

It is well known that the Möbius function of a boolean algebra is \pm 1 , the sign depending only on rank. This yields the following corollary.

Corollary 4.5. If $\sigma(\tau)=1$ and the pair $(\langle\sigma\rangle, \tau)$ is separated, then $\mu(\sigma, \tau)=$ $(-1)^{n-k}$, where $k$ and $n$ are the ranks of $\sigma$ and $\tau$, respectively. 
It should be noted that $[\sigma, \tau]$ can be boolean even if $\sigma(\tau)>1$. For example, the intervals $[123,1324]$ and $[12,3412]$ are both boolean, even though $123(1324)=12(3412)=2$. It would thus be interesting to characterize boolean intervals.

In Section 3, pairs $(\langle\sigma\rangle, \tau)$ with interval blocks were analyzed and shown to satisfy $\mu(\langle\sigma\rangle, \tau)=0$. Moreover, an interval $[\sigma, \tau] \subset \mathcal{P}$ where the pair $(\sigma, \tau)$ has an interval block satisfies $\mu(\sigma, \tau)=0$. Now we examine situations where $\sigma \leq \tau$ in $\mathcal{P}$ and there is an occurrence $\langle\sigma\rangle$ such that the pair $(\langle\sigma\rangle, \tau)$ is interval free. Under certain conditions, the Möbius function for such a poset has the nice form $\mu(\langle\sigma\rangle, \tau)=(-1)^{r}$, where $r$ is the rank of $[\langle\sigma\rangle, \tau]$. We believe that this property holds for a large class of intervals and give one such subclass here.

A few preliminaries are necessary before proving Theorem 4.11.

Definition 4.6. The subposet $\llbracket\langle\pi\rangle, \rho \rrbracket$ of $[\langle\pi\rangle, \rho]$ consists of all $\omega \in[\langle\pi\rangle, \rho]$ such that the pair $(\langle\pi\rangle, \omega)$ is interval free. In particular, it is always the case that $\langle\pi\rangle \in \llbracket\langle\pi\rangle, \rho \rrbracket$, and $\rho \in \llbracket\langle\pi\rangle, \rho \rrbracket$ if the pair $(\langle\pi\rangle, \rho)$ is interval free.

Remark 4.7. By Theorem 3.1, the poset $\llbracket\langle\sigma\rangle, \tau \rrbracket$ is obtained by removing from $[\langle\sigma\rangle, \tau]$ only elements $\pi$ with $\mu(\langle\sigma\rangle, \pi)=0$. When the pair $(\langle\sigma\rangle, \tau)$ is interval free, we are thus replacing $[\langle\sigma\rangle, \tau]$ by a subposet, $\llbracket\langle\sigma\rangle, \tau \rrbracket$, which has the same Möbius function as $[\langle\sigma\rangle, \tau]$.

Definition 4.8. A subset $S$ of a graded poset $P$ has the rank property if it contains as many elements of even rank in $P$ as it does of odd rank. Such an $S$ is called RP. If $S$ is all of $P$ we use the same terminology for $P$ itself.

The following lemma suggests the relevance of the rank property.

Lemma 4.9. An RP poset $P$ has the following properties.

(a) If $X \subseteq P$ is $\mathrm{RP}$ as well, then $P \backslash X$ is $\mathrm{RP}$.

(b) If $P$ is bounded, with minimal element $\hat{0}$ and maximal element $\hat{1}$, and if $\mu_{P}(\hat{0}, x)=(-1)^{\mathrm{rk}(x)}$ for all $x \in[\hat{0}, \hat{1})$, then $\mu_{P}(\hat{0}, \hat{1})=(-1)^{\mathrm{rk}(P)}$.

Proof. Part (a) is obvious.

By Definition 2.6,

$$
\mu(\hat{0}, \hat{1})=-r_{0}+r_{1}-r_{2}+\cdots-(-1)^{\mathrm{rk}(P)-1} r_{\mathrm{rk}(P)-1},
$$

where $r_{i}$ is the number of elements of rank $i$ in $P$. The poset $P$ is RP, so

$$
r_{0}+r_{2}+r_{4}+\cdots=r_{1}+r_{3}+r_{5}+\cdots
$$


with one sum going to $r_{\mathrm{rk}(P)-1}$ and the other to $r_{\mathrm{rk}(P)}$. This is equivalent to

$$
(-1)^{\mathrm{rk}(P)} r_{\operatorname{rk}(P)}=-r_{0}+r_{1}-r_{2}+\cdots-(-1)^{\mathrm{rk}(P)-1} r_{\mathrm{rk}(P)-1} .
$$

Thus equation (5) simplifies to $\mu(\hat{0}, \hat{1})=(-1)^{\mathrm{rk}(P)} r_{\mathrm{rk}(P)}$. Since $r_{\mathrm{rk}(P)}=1$, this means that $\mu(\hat{0}, \hat{1})=(-1)^{\mathrm{rk}(P)}$, proving part (b).

We will show that under particular conditions, the poset $\llbracket\langle\sigma\rangle, \tau \rrbracket$ is RP, regardless of whether the pair $(\langle\sigma\rangle, \tau)$ is interval free. When the pair is interval free, and thus $\tau \in \llbracket\langle\sigma\rangle, \tau \rrbracket$, this will imply that $\mu(\langle\sigma\rangle, \tau)=(-1)^{n-k}$ in both $[\langle\sigma\rangle, \tau]$ and $\llbracket\langle\sigma\rangle, \tau \rrbracket$.

Recall Definition 2.14 of the set $C=\tau \backslash\langle\sigma\rangle$ : $C$ consists of those values of $[1, n]$ that are not part of the occurrence $\langle\sigma\rangle$. This set holds the key to the proof because we have fixed the occurrence $\langle\sigma\rangle$ of $\sigma$ in $\tau$, so elements of $[\langle\sigma\rangle, \tau]$ differ only in the letters of $C$.

Any permutation $\rho \in[\langle\sigma\rangle, \tau]$ can be identified with the subset of letters $\rho \backslash\langle\sigma\rangle \subseteq C$. However, there may be distinct subsets $S$ and $S^{\prime}$ of $C$ for which the permutations $\langle\sigma\rangle+S$ and $\langle\sigma\rangle+S^{\prime}$ are order isomorphic, having the occurrence $\langle\sigma\rangle$ in the same places. Of course, two such permutations correspond to the same element of $[\langle\sigma\rangle, \tau]$. When discussing something of the form $\langle\sigma\rangle+S$, where the letters of $S$ are added to the occurrence $\langle\sigma\rangle$, it will be understood that this indicates the order isomorphic element in $[\langle\sigma\rangle, \tau]$ (or in $\llbracket\langle\sigma\rangle, \tau \rrbracket$ as appropriate), having the occurrence $\langle\sigma\rangle$ in the same positions.

We now come to the definitions of the conditions under which the Möbius function of an interval free pair $(\sigma, \tau)$ can be shown to be either 1 or -1 , depending only on the rank of the interval. Note that there is a relationship between the following definition and the notion of "separated" (see Definition 4.2).

Definition 4.10. Two letters $a$ and $b$, with $a<b$, in the complement of $\sigma$ in $\tau$ are similar if they are in the same region and there is no $c \in\langle\sigma\rangle$ such that $a<c<b$. A group of similar letters in the complement consists of two or more similar letters.

As an example, if $\tau=357128469$ and $\langle\sigma\rangle=\langle 184\rangle$, then the regions are $357-2-69$. The letters 3 and 5 are not similar, since $4 \in\langle\sigma\rangle$. The letters 5 and 7 are similar, because the only letter between them in value, namely 6 , belongs to the complement of $\langle\sigma\rangle$.

Theorem 4.11. Fix $\sigma \in S_{k}$ and $\tau \in S_{n}$, where $k\langle n$, and let $\langle\sigma\rangle$ be an occurrence of $\sigma$ in $\tau$. If there is at most one maximal group of similar letters in all the regions of $\tau$, then $\llbracket\langle\sigma\rangle, \tau \rrbracket$ is $\mathrm{RP}$. 
Proof. Suppose that there is no maximal group; that is, no two letters in the same region are similar. Then the pair $(\sigma, \tau)$ is separated, so, by Theorem 4.4, the interval $\llbracket\langle\sigma\rangle, \tau \rrbracket$ is a nontrivial boolean algebra, which is RP.

Now suppose that there is a unique maximal group of similar letters, and that it consists of $m \geq 2$ letters. We prove the result by induction on $(n-k)-m$.

If $n-k=m$, then the entire complement $\tau \backslash\langle\sigma\rangle$ is this group of $m$ similar letters, which forms an interval block. Thus $\llbracket\langle\sigma\rangle, \tau \rrbracket$ consists of two elements: the element $\langle\sigma\rangle$ and the element obtained by adding to $\langle\sigma\rangle$ a single letter from the complement $\tau \backslash\langle\sigma\rangle$. This poset is obviously RP.

Now assume the result for all $(n-k)-m<r$, where $r \geq 1$ and suppose that $(n-k)-m=r$. This means that in addition to the group of $m$ elements, there are $r$ other letters in $\tau \backslash\langle\sigma\rangle$, none of which is similar to any other letter in its respective region. Suppose that $x$ is one of these letters. Then the poset $\llbracket\langle\sigma\rangle, \tau \rrbracket$ is isomorphic to the disjoint union of the posets $\llbracket\langle\sigma\rangle, \tau \backslash\{x\} \rrbracket \sqcup \llbracket\langle\sigma\rangle+x, \tau \rrbracket$, based on whether an element of $\llbracket\langle\sigma\rangle, \tau \rrbracket$ includes the letter $x$ or not. Each of these intervals satisfies the hypotheses of the theorem, and by induction we know that they are both RP. Therefore $\llbracket\langle\sigma\rangle, \tau \rrbracket$ is $\mathrm{RP}$ as well.

Corollary 4.12. If $\sigma$ and $\tau$ satisfy the hypotheses of Theorem 4.11, and the pair $(\sigma, \tau)$ is interval free, then $\mu(\sigma, \tau)=(-1)^{n-k}$.

Proof. This follows from Lemma 4.9 and Theorem 4.11.

The hypotheses of Theorem 4.11 cannot be weakened to encompass all interval free pairs $(\sigma, \tau)$. The smallest $\tau$ for which there are counterexamples $[\langle\sigma\rangle, \tau]$ with $\mu(\sigma, \tau) \neq \pm 1$ have length 10 , where the shortest $\sigma$ have length 3 . The only counterexample in that case, up to trivial symmetries, is when $\sigma=321$ and $\tau=2,5,1,7,3,10,4,6,9,8$ (we use commas here to clarify which values are two-digit numbers). The only occurrence of $\sigma$ in $\tau$ here is $\langle 10,9,8\rangle$, so the complement is $25173-46$, which has no interval blocks, but $\mu(\sigma, \tau)=0$.

Another counterexample, where the Möbius function has value 2, is

$$
\sigma=2341, \quad \tau=2,3,8,1,6,12,4,10,5,9,7,11,2 .
$$

Here the only occurrence of 2341 is $\langle 2381\rangle$. Thus the complement is a single region of eight letters. 


\section{Open problems}

We have gathered data to support the following two conjectures.

Conjecture 5.1. Suppose that $\tau$ avoids the pattern 132 (or, equivalently, 312 , or 213 , or 231$)$. Then $\mu(1, \tau)$ is either 0,1 , or -1 .

Conjecture 5.2. Suppose that $\tau$, and hence the entire interval $[\sigma, \tau]$ for any $\sigma$, avoids the pattern 132 (or, equivalently, 312, or 213, or 231). Then we have $|\mu(\sigma, \tau)| \leq \sigma(\tau)$.

Because of the symmetries between the patterns 132, 231, 213, 312, if Conjecture 5.2 is true for any one of them, it is true for each of them. Thus, the combined contrapositives of the corresponding four conjectures say that if $|\mu(\sigma, \tau)|$ exceeds $\sigma(\tau)$, then $\tau$ must contain all of the patterns 132, 231, 213 and 312 .

Observe that if Conjecture 5.2 holds, then, for 132-avoiding intervals $[\sigma, \tau]$ where $\sigma$ occurs precisely once in $\tau$, the Möbius function must be 0,1 or -1 .

Additionally, recalling Section 4, it would be interesting to characterize the boolean intervals in the poset of permutation patterns.

\section{Acknowledgment}

We are indebted to Eric Babson for very helpful discussions, and to an anonymous referee for many valuable suggestions.

\section{References}

[1] M. H. Albert, M. D. Atkinson, and M. Klazar, The enumeration of simple permutations, J. Integer Seq. 6 (2003), 03.4.4. MR2051958

[2] R. Brignall, A survey of simple permutations, arXiv:0801.0963v2.

[3] B. E. Sagan and V. Vatter, The Möbius function of a composition poset, J. Algebraic Combin. 24 (2006), 117-136. MR2259013

[4] R. P. Stanley, Enumerative Combinatorics, vol. 1, Cambridge Studies in Advanced Mathematics, no. 49, Cambridge University Press, Cambridge, 1997. MR1442260

[5] H. Wilf, The patterns of permutations, Discrete Math. 257 (2002), 575583. MR1935750 
EinAR SteingrímSSON

The Mathematics Institute

School of Computer ScIEnce

REYKJAVÍK UNIVERSITY

ICELAND

E-mail address: einarst@ru.is

Bridget Eileen Tenner

Department of Mathematical Sciences

DePaul University

Chicago, Illinois

USA

E-mail address: bridget@math.depaul.edu

ReCEIVED January 15, 2010 